\title{
Quantum-Limited Time-Frequency Estimation through Mode-Selective Photon Measurement
}

\author{
J. M. Donohue, ${ }^{1, *}$ V. Ansari, ${ }^{1}$ J. Řeháček, ${ }^{2}$ Z. Hradil, ${ }^{2}$ B. Stoklasa, ${ }^{2}$ M. Paúr, ${ }^{2}$ L. L. Sánchez-Soto,,${ }^{3,4}$ and C. Silberhorn ${ }^{1}$ \\ ${ }^{1}$ Integrated Quantum Optics, Paderborn University, Warburger Strasse 100, 33098 Paderborn, Germany \\ ${ }^{2}$ Department of Optics, Palacký University, 17. listopadu 12, 77146 Olomouc, Czech Republic \\ ${ }^{3}$ Departamento de Óptica, Facultad de Física, Universidad Complutense, 28040 Madrid, Spain \\ ${ }^{4}$ Max-Planck-Institut für die Physik des Lichts, Staudtstrasse 2, 91058 Erlangen, Germany
}

(Received 11 May 2018; published 27 August 2018)

\begin{abstract}
By projecting onto complex optical mode profiles, it is possible to estimate arbitrarily small separations between objects with quantum-limited precision, free of uncertainty arising from overlapping intensity profiles. Here we extend these techniques to the time-frequency domain using mode-selective sumfrequency generation with shaped ultrafast pulses. We experimentally resolve temporal and spectral separations between incoherent mixtures of single-photon level signals ten times smaller than their optical bandwidths with a tenfold improvement in precision over the intensity-only Cramér-Rao bound.
\end{abstract}

DOI: 10.1103/PhysRevLett.121.090501

Introduction.-The time-honored Cramér-Rao lower bound (CRLB) [1,2] is credibly the most appropriate tool to address the resolution limits for incoherent imaging, as highlighted in recent years [3-9]. This is especially pertinent when photon shot noise is the dominant noise source (as in, e.g., astronomical observations) and a statistical treatment of resolution is indispensable. Nonetheless, in spite of these compelling results, Cramér-Rao resolution limits did not demand a great deal of attention until recent works examined microscopy limitations from a photon-counting perspective [10-12]. The chief idea can be formalized through the Fisher information [13], which quantifies the amount of information gained per photon detection and is directly associated to the CRLB. For direct intensity imaging, the Fisher information drops to zero for object separations smaller than the spread of the optical field. This precipitous drop, named Rayleigh's curse, limits the usefulness of photon counting for metrology.

This line of questioning cleared the way for a fresh reexamination of the problem by Tsang and coworkers [14-18]. Surprisingly, when one calculates the quantum Fisher information [19] (i.e., optimized over all the possible quantum measurements), the associated quantum CRLB maintains a fairly constant value for any separation of the sources. This shows the potential for parameter estimation of distributions with precision unaffected by Rayleigh's curse. The key behind these techniques is phase-sensitive measurement in mode bases other than intensity $[20,21]$, requiring more elegant measurement procedures but no fragile quantum resources. This has been experimentally demonstrated for spatially separated objects by holographic mode projection [22], heterodyne detection [23,24], and parity-sensitive interferometers [25]. As the space-time duality of light has already provided valuable insights and tools in classical time-frequency measurement [26-28], it is worthwhile to consider the advantages these techniques can offer when adapted to metrology and analysis in the time-frequency domain [29-32].

In this Letter, we show that mode-selective measurement can be harnessed to estimate separations in time and frequency well below the spread of the source light. In an analogy to the Rayleigh limit in space, this allows us to overcome the equivalent criterion in measuring spectral separations [33], which states that the minimum resolvable separation of the spectral maxima is equal to the halfmaximum width. We experimentally realize this enhancement in both time and frequency estimation settings by projectively measuring Hermite-Gauss time-frequency modes, as initially proposed in Ref. [34]. We extend this idea to click-counting detection using sum-frequency generation with shaped ultrafast pulses in group-velocity engineered nonlinear waveguides. We explicitly demonstrate precision below the intensity-only CRLB, establishing mode-selective measurement as a valuable tool for pushing metrological limits in multiple physical domains.

Quantum analysis of time-frequency metrological problems has already provided a plethora of useful tools. In particular, quantum advantages can be realized in time-of-flight measurement and synchronization by exploiting entanglement [35], squeezing [34,36], and bunching [37], and considering quantum techniques and analysis has inspired classical techniques that outperform their preexisting counterparts [38]. Additionally, enhanced time-of-flight ranging techniques with quantum limits outperforming intensity detection have been demonstrated using homodyne detection with shaped local oscillators in higher-order Hermite-Gaussian modes $[34,36,39,40]$. Here, we show that quantum-inspired metrology finds application 
in measuring incoherent source superpositions with either time or frequency offsets using tailored nonlinear optical interactions and click-counting detection. The tools used herein reach the quantum limit with single-photon level signals, but can be described and realized in a completely classical fashion, requiring no quantum resources and having equal applicability to quantum and classical signals. This form of frequency estimation has natural applications in, e.g., measuring nearly degenerate atomic and stellar spectral lines, particularly after undergoing inhomogeneous broadening. Precision time measurements find natural applications in time-of-flight ranging and in probing ultrafast system dynamics.

Quantum-limited measurements.-We formalize the parameter estimation problem under consideration analogously to the spatial case $[14,22]$. Two mutually incoherent (or phase-randomized) light sources with equal intensities emit at optical frequencies $\nu_{0} \pm \mathfrak{G}_{\nu} / 2$. We assume that the central frequency $\nu_{0}$ is well known and that the remaining quantity of interest is the spectral separation, $\mathfrak{B}_{\nu}$. If the sources have non-negligible spectral bandwidth, the optical spectrum $I\left(\nu, \mathfrak{g}_{\nu}\right)$ of the incoherent mixture as measured on a spectrometer will be

$$
I\left(\nu, \mathfrak{g}_{\nu}\right)=\frac{1}{2}\left[\left|\psi\left(\nu+\frac{\mathfrak{g}_{\nu}}{2}\right)\right|^{2}+\left|\psi\left(\nu-\frac{\mathfrak{g}_{\nu}}{2}\right)\right|^{2}\right],
$$

where $\psi(\nu)$ is the spectral amplitude shape. For specificity, we focus on the case of Gaussian spectral amplitudes (frequency-domain point-spread functions) with rootmean-square (rms) widths $\sigma_{\nu}$, such that

$$
\psi\left(\nu \pm \frac{\mathfrak{g}_{\nu}}{2}\right)=\frac{1}{\left(2 \pi \sigma_{\nu}^{2}\right)^{1 / 4}} \exp \left(-\frac{\left(\nu-\nu_{0} \pm \frac{\mathfrak{g}_{\nu}}{2}\right)^{2}}{4 \sigma_{\nu}^{2}}\right) .
$$

The standard method of estimating the spectral separation $\mathfrak{g}_{\nu}$ in the low-luminescence (i.e., photon counting) regime would be to measure the spectral intensity $I\left(\nu, \mathfrak{S}_{\nu}\right)$ on a spectrometer, such as a Fabry-Perot interferometer or grating-based spectrograph, and use a fitting or deconvolution algorithm on the integrated photon counts. We quantify the amount of information in principle available to estimate $\mathfrak{g}_{\nu}$ with $N$ detected photons (i.e., standard intensity click-counting detection) via the Fisher information $\mathcal{F}_{\text {std }}$, given by

$$
\mathcal{F}_{\text {std }}=N \int_{-\infty}^{\infty} d \nu \frac{1}{I\left(\nu, \mathfrak{g}_{\nu}\right)}\left(\frac{\partial I\left(\nu, \mathfrak{g}_{\nu}\right)}{\partial \mathfrak{\Xi}_{\nu}}\right)^{2} .
$$

The Fisher information quantifies how sensitive the measured quantity $I\left(\nu, \mathfrak{S}_{\nu}\right)$ is to changes in the variable $\mathfrak{g}_{\nu}$, and can be used to construct the CRLB as $\operatorname{Var}\left(\hat{\mathfrak{\Xi}}_{\nu}\right) \geq 1 / \mathcal{F}_{\text {std }}$ [9], which defines the absolute minimum mean-squared error (variance) of the estimated separation, $\hat{\mathfrak{\xi}}_{\nu}$. For large separations, $\mathfrak{g}_{\nu} \gg \sigma_{\nu}$, the standard Fisher information is constant, providing a Cramér-Rao bounded variance of $\operatorname{Var}\left(\hat{\mathfrak{\xi}}_{\nu}\right) \geq\left(4 \sigma_{\nu}^{2}\right) / N$. However, when $\mathfrak{g}_{\nu} \sim \sigma_{\nu}$, the CRLB bound grows dramatically, diverging as $\mathfrak{g}_{\nu} / \sigma_{\nu}$ approaches zero. This behavior is known as Rayleigh's curse in the spatial domain, and is sometimes rephrased as the Taylor criterion in spectral measurements. Note that the exact same "curse" applies to estimating incoherent time separations, $\mathfrak{g}_{t}$, between two pulsed sources through direct timing measurement, e.g., with autocorrelation or streakcamera techniques [41].

The curse can be lifted by performing phase- or paritysensitive measurements, even though the source fields themselves have no coherent phase relationship. An optimal measurement basis is always provided by the partial derivatives of the amplitude point-spread function [21]. For the Gaussian point-spread function as in Eq. (2), the optimal measurement is then the Hermite-Gaussian basis [21,22]. For separations $\mathfrak{g}_{\nu} \lesssim \sigma_{\nu}, \mathfrak{g}_{\nu}$ can be estimated with only projections onto the first two Hermite-Gauss modes, expressed as

$$
\begin{aligned}
& \phi_{\wedge}(v)=\frac{1}{\left(2 \pi \sigma_{v}^{2}\right)^{\frac{1}{4}}} \exp \left(-\frac{\left(v-v_{0}\right)^{2}}{4 \sigma_{v}^{2}}\right) \\
& \phi \backsim(v)=\frac{\left(v-v_{0}\right)}{\left(2 \pi \sigma_{v}^{6}\right)^{\frac{1}{4}}} \exp \left(-\frac{\left(v-v_{0}\right)^{2}}{4 \sigma_{v}^{2}}\right) .
\end{aligned}
$$

If projective measurements onto these modes can be realized, the estimator $\hat{\mathfrak{\xi}}_{\nu}$ has a curse-free performance, with $\operatorname{Var}\left(\hat{\mathfrak{\vartheta}}_{\nu}\right) \geq\left(4 \sigma_{\nu}^{2}\right) / N$ for arbitrarily small values of $\mathfrak{\mathfrak { g }}_{\nu}$. This value agrees exactly with the absolute quantum limit derived from the quantum Fisher information [14]. To include estimation of the centroid, extend the technique to large separations $\mathfrak{G}_{\nu} \gg \sigma_{\nu}$, or, in cases with unequalintensity emission, higher-order mode projections may be used to maintain an advantage over standard intensity measurements [42].

Time-frequency mode selection.-The key experimental requirement to enable this advantage is mode-selective projective measurement in the time-frequency domain. We implement such measurements using a technique known as the quantum pulse gate [43-47], a sum-frequency process where a weak input signal is mixed with a spectrally shaped pump pulse to create an upconverted signal in a long, nonlinear waveguide. To implement a quantum pulse gate, the input signal and pump pulses must have matched group velocities and the walkoff between the input and upconverted signals must be longer than the length of the input pulses. If these conditions are met, the probability of an upconversion event in the low-efficiency regime given an input spectral amplitude $\psi(\nu)$ and a pump amplitude $\alpha(\nu)$ can be expressed simply as $[43,47]$

$$
P_{\alpha} \propto\left|\int d \nu \alpha(-\nu) \psi(\nu)\right|^{2} .
$$


Measuring the upconverted pulse power thereby corresponds to a projective measurement on the broadband timefrequency mode defined by the shape of the pump pulse, $\alpha^{*}(-\nu)$ [46]. By counting photons in the upconverted mode while projecting on either the fundamental Gaussian mode or the first-order Hermite-Gaussian mode, we can easily construct an estimator by taking their ratio.

The mode selectivity of the quantum pulse gate is limited by the group-velocity walkoff between the input and upconverted signals, which we define by the walkoff parameter $\Delta=L / 2\left(1 / u_{\text {in }}-1 / u_{\text {out }}\right)$, where $u_{j}$ is the group velocity and $L$ is the length of the nonlinear interaction. This walkoff defines the phase matching conditions of the interaction, imposing a rms bandwidth of the upconverted light of $\sigma_{\mathrm{PM}} \approx 0.18 / \Delta$. When the input and pump are significantly broader than the phase matching bandwidth and the side lobes arising from the sinc-shaped phase matching curve are filtered out, the ratio of the lowest-order Hermite-Gaussian projections is given by

$$
\frac{P}{P_{\wedge}} \approx \frac{\sigma_{\mathrm{PM}}^{2}}{4 \sigma_{v}^{2}}+\frac{\mathbb{8}_{t}^{2}}{16 \sigma_{t}^{2}}+\frac{\mathbb{B}_{v}^{2}}{16 \sigma_{v}^{2}},
$$

where $\sigma_{t}$ and $\sigma_{\nu}$ are the rms widths of the measurement pulse's temporal and spectral profiles; a derivation of this result is presented in the Supplemental Material [48]. If the signal is properly aligned in one of the 2 degrees of freedom $\left(\mathfrak{g}_{\nu}=0\right.$ or $\left.\mathfrak{g}_{t}=0\right)$ and $\mathfrak{I}_{\nu} / \sigma_{\nu} \gg \sigma_{\mathrm{PM}} / \sigma_{\nu}$ or $\mathfrak{I}_{t} / \sigma_{t} \gg \sigma_{\mathrm{PM}} / \sigma_{\nu}$, Eq. (6) shows that the square root of the ratio of projection probability for the first two Hermite-Gauss modes can be used as an exact estimator for the separation between the signals. For separations small enough that the finite phase matching bandwidth cannot be completely neglected, Eq. (6) can still be inverted to construct an estimator $\hat{\mathfrak{\xi}}_{\nu}$ or $\hat{\mathfrak{s}}_{t}$, although with slightly reduced precision relative to the quantum limit. As the phase matching bandwidth can be much smaller than the input bandwidths [49], the precision of this method can be considerably finer than the broad bandwidth or temporal durations of the pulses being interrogated.

Experiment.-In our experimental apparatus, sketched in Fig. 1, we generate shaped input signal and pump pulses from a Ti:sapphire laser and optical parameter oscillator (OPO) with a repetition rate of $80 \mathrm{MHz}$. The strong pump pulses at $875 \mathrm{~nm}$ are shaped into Hermite-Gauss modes with a bandwidth of $1.3 \mathrm{~nm}$ full width at half maximum (FWHM) using a $4 f$ line with a spatial light modulator (SLM) at the focal plane, with approximately $2 \mathrm{~mW}$ coupled into the quantum pulse gate.

To create frequency- and time-shifted pulses, we carve Gaussian signals with intensity rms widths of $\sigma_{\nu}=$ $182 \pm 2 \mathrm{GHz}$ from the approximately $3 \mathrm{THz}$ FWHM emission of the OPO using a commercial pulse shaper (Finisar 4000S). Frequency shifts are imparted straightforwardly by carving different parts of the OPO spectrum,

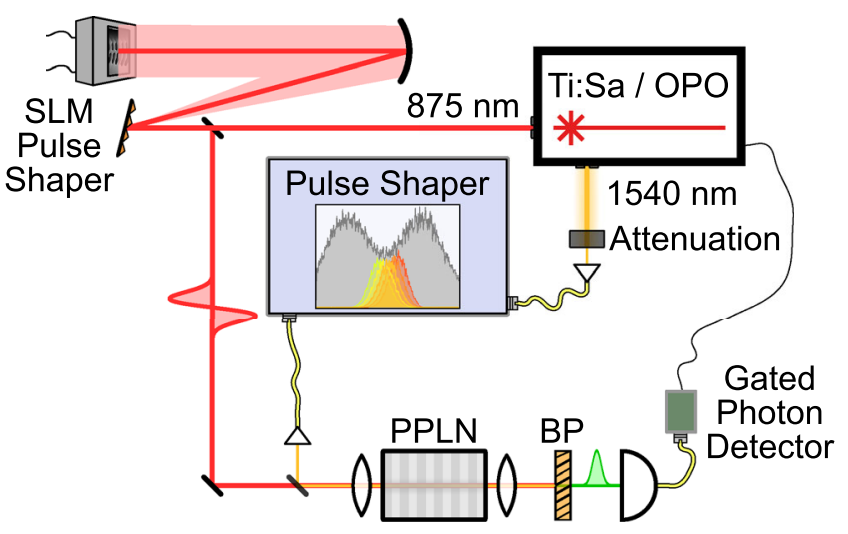

FIG. 1. Experimental setup.-We carve signal pulses with shifting center frequencies and time delays from an attenuated broadband Ti:sapphire OPO pulse at $1540 \mathrm{~nm}$ using a commercial telecommunications pulse shaper. We shape pump pulses at $875 \mathrm{~nm}$ into Hermite-Gaussian shapes using a $4 f$ line with a spatial light modulator (SLM). We then mix the pump and signal pulses in a PPLN waveguide, separate the sum-frequency signal with a $4 f$ bandpass filter (BP), and count photons using an avalanche photodiode gated by a clock pulse from the Ti: sapphire.

while time shifts are imparted by programming linear spectral phases with the pulse shaper. The width of the pulses in time was measured to be $\sigma_{t}=387 \pm 13$ fs using the quantum pulse gate as an autocorrelator. Neutral density filters were used to attenuate the shaped pulses to approximately 1.1 photons per pulse coupled into the measurement waveguide. The incoherence of the time- and frequencyseparated mixtures was assured by switching between positive and negative shifts and mixing the measured results.

The quantum pulse gate was realized by combining the shaped input and pump pulses on a dichroic mirror and coupling them into a $17 \mathrm{~mm}$-long and $7-\mu \mathrm{m}$-wide periodically poled lithium niobate (PPLN) waveguide heated to $470 \mathrm{~K}$ with a poling period of $4.4 \mu \mathrm{m}$ and single-mode propagation at $1540 \mathrm{~nm}$. The spectra of the upconverted light at $558 \mathrm{~nm}$ was cleaned with a $4 f$ line to remove phase matching side lobes, resulting in an upconverted bandwidth of $\sigma_{\mathrm{PM}}=28 \mathrm{GHz}$, a factor of 6 smaller than the input light. To reduce background noise due to detector dark counts, the upconverted signal was measured with an avalanche photodiode in coincidence with a clock pulse from the Ti: sapphire sampled down by a factor of 50, resulting in an effective experimental repetition rate of $1.6 \mathrm{MHz}$.

Results and discussion.-Twenty separations ranging from $0-2 \sigma$ were programmed in both time and frequency during the experiment and each setting was measured 60 times. In addition to controlling the separation, the pulse shaper was also used to attenuate the weak input signal to $100 \%, 50 \%$, or $25 \%$ of its original intensity, to demonstrate the lack of any bias due to background noise. The uncorrected estimator $\hat{\mathrm{z}}=4 \sqrt{P / P_{A}}$ from Eq. (6) is shown in Fig. 2. The estimator is seen to reach the expected linear 

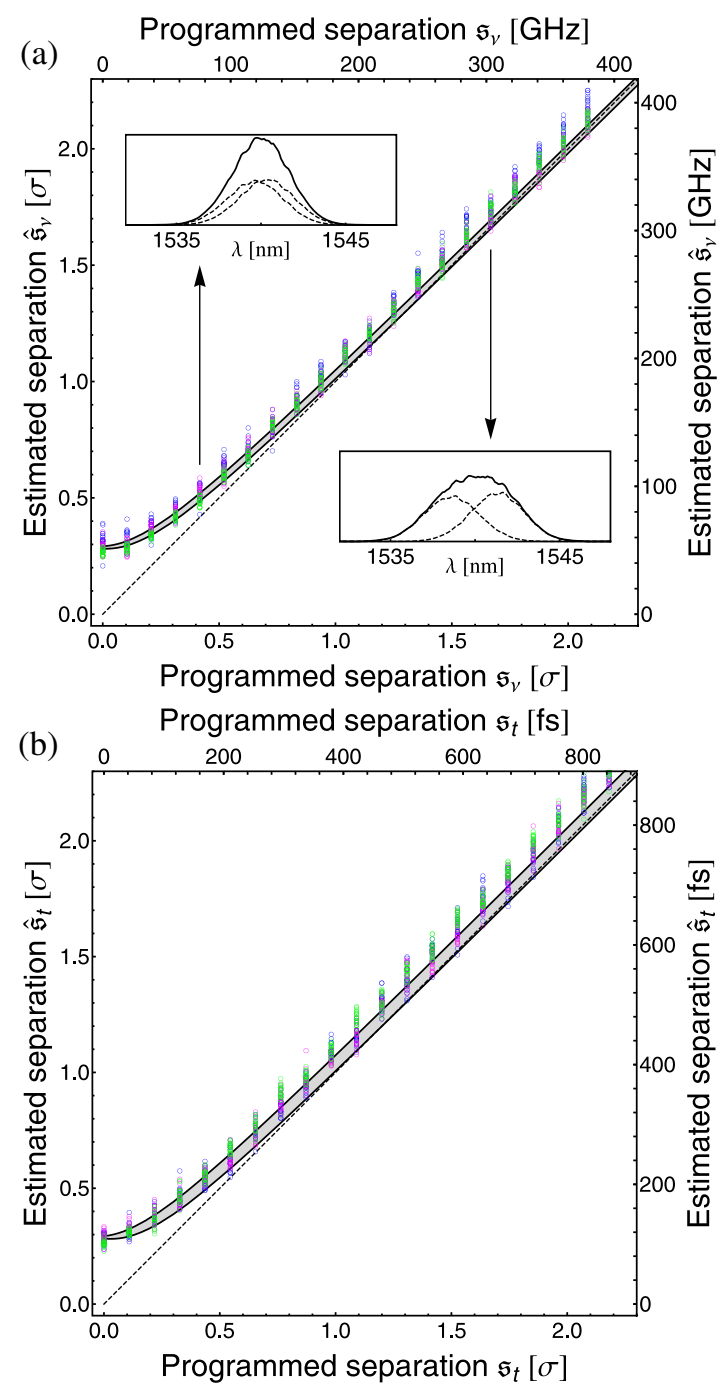

FIG. 2. Raw estimator from time-frequency mode selection.The estimator calculated from the measured counts when mixing frequency- or time-shifted pulses is shown above, in (a) and (b) respectively. The solid black lines correspond to the theoretical expectation given the measured phase matching bandwidth, and the dashed line to the ideal slope-one estimator. The error in the theory curves corresponds to instrument setting and bandwidth characterization uncertainty. In both cases, limitations are encountered for separations below $0.2 \sigma$, as expected from the mode selectivity of the device. The insets on the frequencymeasurement plot (a) provide the spectra of the individually shifted signals (dashed) and their incoherent mixture (solid) for programmed separations of $\mathfrak{g}_{\nu}=0.42 \sigma$ and $1.67 \sigma$.

behavior for separations on the same order as the rms widths, but the imperfect mode selectivity causes small, predictable deviations for very small separations. The observed extinction ratio between the first- and zeroth-order Hermite-Gauss mode when no separation is programmed is found to be $-10 \log _{10}\left(P / P_{\wedge}\right)=(22.9 \pm 0.3) \mathrm{dB}$, corresponding to a minimum estimator value of $\hat{\mathfrak{s}}_{\text {min }}=0.144 \pm 0.005$.

To construct an unbiased estimator resilient to the imperfect selectivity of our device, we use calibration data

from projections onto the first three Hermite-Gauss modes to perform measurement tomography of our technique. Details on the tomography techniques are presented in the Supplemental Material [48]. To demonstrate the precision of our technique, in Fig. 3, we show the variance of the calibrated estimator $\operatorname{Var}(\hat{\mathfrak{\mathfrak { Z }}})$ for both time and frequency measurements while varying the total number of detection events, alongside the standard and quantum CRLBs for the same number of detected photons. The variance is above the quantum limit (in red), owing to mode-selectivity limitations and instabilities. However, it remains below the intensity-only bound $1 / \mathcal{F}_{\text {std }}$ for separations well below the point-spread function widths, with an improvement in precision by a factor of as high as ten for small separations.

The CRLB shown in Fig. 3 is based on the total number of detected photons, effectively postselecting on successful upconversion and neglecting lost photons. If we assume the loss is uniformly distributed across all inputs and projections, the advantage over intensity measurement per photon counted remains valid. We estimate the efficiency of our measurement apparatus by projecting onto the fundamental Gaussian mode for a signal with no temporal or spectral

(a)
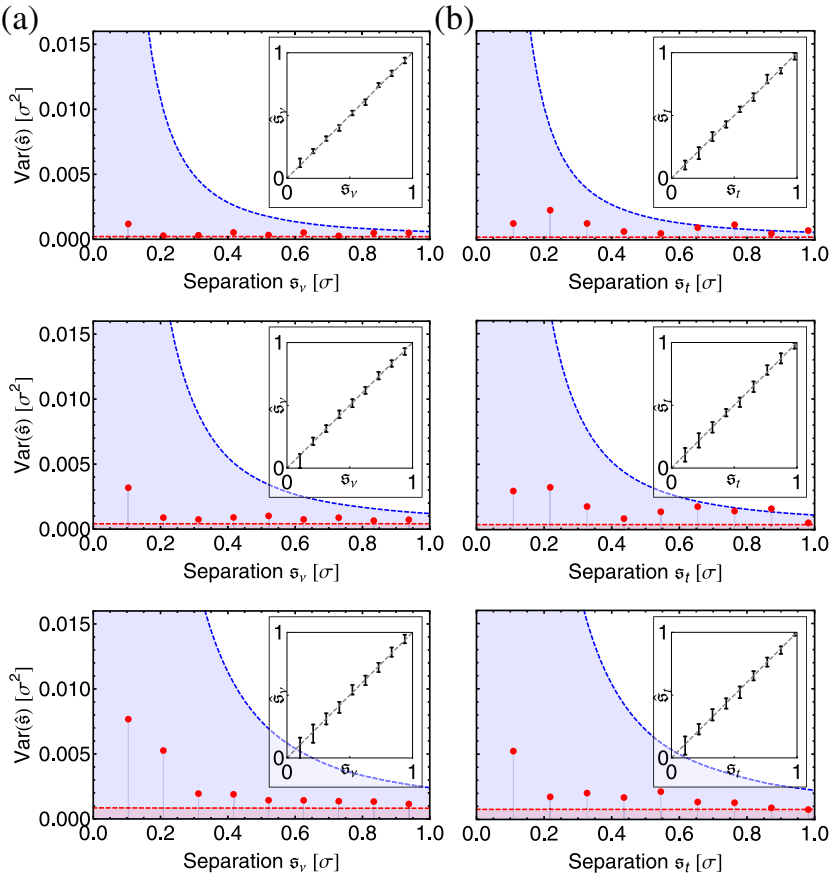

(b)
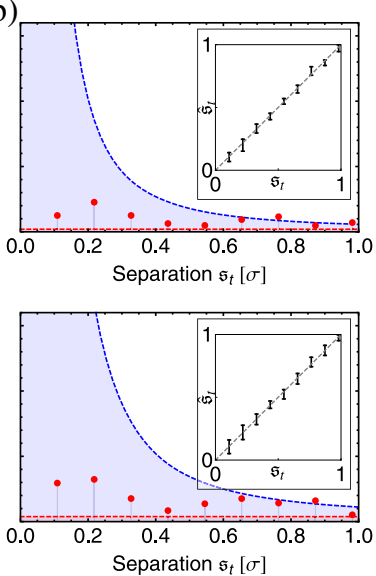

FIG. 3. Variance of the estimator against the standard bound.-The variance (mean-squared error) of the estimator $\hat{\mathfrak{G}}$ as the (a) frequency separation $\mathfrak{g}_{\nu}$ or (b) time separation $\mathfrak{g}_{t}$ is increased. The photon-counting measurements consist of a total of 20000,10000 , and 5000 detection events, from top to bottom. The blue-filled area corresponds to the CRLB for standard intensity detection, and the red dashed line to the quantum limit for the same number of detected photons. Red data points correspond to the variance of the estimator after measurement tomography. The inset shows the estimator after measurement tomography. 
offset. The overall efficiency, including upconversion, spectral isolation, fiber coupling, and photon detection, is approximately $0.7 \%$. From the depletion of the $1540 \mathrm{~nm}$ signal, we isolate the efficiency of the upconversion process as approximately 18\%. Notably, no sources of loss are fundamental to the principle of our technique. Photon detection and coupling loss are factors in both our scheme and direct intensity detection. The spectral isolation of the upconverted signal can be made redundant through domain engineering of the nonlinear medium, removing (apodizing) the side lobes of the sinc-shaped phase matching function [50]. Finally, it is possible to reach unit upconversion efficiency while maintaining mode selectivity using multiple-stage versions of the quantum pulse gate [51].

The above results clearly demonstrate that mode-selective time-frequency measurement can be exploited for precision parameter estimation problems where intensity measurements fail. Notably, the absolute time and frequency scales accessible are not strongly dependent on the scale of the measurement pulses, but rather the material properties, namely the phase matching bandwidth $\sigma_{\mathrm{PM}}$. In our realization, this corresponded to time and frequency scales of $200 \mathrm{fs}$ and $100 \mathrm{GHz}$, respectively. The accessible time and frequency scales could be improved either along with the conversion efficiency by increasing the nonlinear interaction length, or at the expense of the detection rate through narrow-band filtering of the upconverted signal. Alternative methods based on mode-selective atomic or solid-state Raman memories could provide greater sensitivity, particularly in the frequency domain $[52,53]$. Techniques based on homodyne detection can also provide the necessary mode selectivity in the time-frequency domain $[34,54,55]$.

We have demonstrated that parameter estimation in the time-frequency domain can benefit greatly from quantuminspired techniques and analysis. By exploiting timefrequency mode-selective measurement enabled by waveguided nonlinear interactions, we have shown that sub-pulse-width separations can be estimated with precision below the standard CRLB. By adapting these techniques to different scales, this method could find immediate practical use in atomic and stellar spectral characterization and timeof-flight imaging. Future work will explore different modeselective systems to adapt to specific tangible metrological problems and apply higher-order projections to multiparameter estimation protocols.

We thank K. Bonsma-Fisher, O. Di Matteo, J. Gil-López, M. Allgaier, C. Fabre, N. Treps, and B. Brecht for fruitful discussions. This research has received funding from the European Unions (EU) Horizon 2020 research and innovation program under Grant Agreement No. 665148, the Grant Agency of the Czech Republic (Grant No. 18-04291S), Palacký University (Grant No. IGA-PrF-2018-003), and the Spanish MINECO (Grant No. FIS2015-67963-P). J. M. D. gratefully acknowledges support from Natural Sciences and Engineering Research Council of Canada. *john.matthew.donohue@uni-paderborn.de

[1] H. Cramér, Mathematical Methods of Statistics (Princeton University Press, Princeton, NJ, 1946).

[2] R. C. Rao, Bull. Calcutta Math. Soc. 37, 81 (1945).

[3] E. J. Farrell, J. Opt. Soc. Am. 56, 578 (1966).

[4] T. Orhaug, Opt. Acta 16, 75 (1969).

[5] C. W. Helstrom, J. Opt. Soc. Am. 60, 233 (1970).

[6] C. W. Helstrom, J. Opt. Soc. Am. 60, 659 (1970).

[7] J. Zmuidzinas, J. Opt. Soc. Am. A 20, 218 (2003).

[8] R. Holmes, B. Calef, D. Gerwe, and P. Crabtree, Appl. Opt. 52, 5235 (2013).

[9] L. Motka, B. Stoklasa, M. D’Angelo, P. Facchi, A. Garuccio, Z. Hradil, S. Pascazio, F. V. Pepe, Y. S. Teo, J. Rehacek, and L. L. Sanchez-Soto, Eur. Phys. J. Plus 131, 130 (2016).

[10] E. Bettens, D. Van Dyck, A. J. den Dekker, J. Sijbers, and A. van den Bos, Ultramicroscopy 77, 37 (1999).

[11] S. Ram, E. Sally Ward, and R. J. Ober, Proc. Natl. Acad. Sci. U.S.A. 103, 4457 (2006).

[12] J. Chao, E. Sally Ward, and R. J. Ober, J. Opt. Soc. Am. A 33, B36 (2016).

[13] R. A. Fisher, Math. Proc. Cambridge Philos. Soc. 22, 700 (1925).

[14] M. Tsang, R. Nair, and X.-M. Lu, Phys. Rev. X 6, 031033 (2016).

[15] R. Nair and M. Tsang, Phys. Rev. Lett. 117, 190801 (2016).

[16] R. Nair and M. Tsang, Opt. Express 24, 3684 (2016).

[17] M. Tsang, New J. Phys. 19, 023054 (2017).

[18] M. Tsang, J. Mod. Opt. 65, 1385 (2018).

[19] D. Petz and C. Ghinea, in Quantum Probability and Related Topics (World Scientific, Singapore, 2011), Vol. 27, pp. 261-281.

[20] C. Lupo and S. Pirandola, Phys. Rev. Lett. 117, 190802 (2016).

[21] J. Rehacek, M. Paúr, B. Stoklasa, Z. Hradil, and L. L. Sánchez-Soto, Opt. Lett. 42, 231 (2017).

[22] M. Paur, B. Stoklasa, Z. Hradil, L. L. Sanchez-Soto, and J. Rehacek, Optica 3, 1144 (2016).

[23] F. Yang, A. Taschilina, E. S. Moiseev, C. Simon, and A. I. Lvovsky, Optica 3, 1148 (2016).

[24] F. Yang, R. Nair, M. Tsang, C. Simon, and A. I. Lvovsky, Phys. Rev. A 96, 063829 (2017).

[25] W. K. Tham, H. Ferretti, and A. M. Steinberg, Phys. Rev. Lett. 118, 070801 (2017).

[26] C. V. Bennett, R. P. Scott, and B. H. Kolner, Appl. Phys. Lett. 65, 2513 (1994).

[27] V. Torres-Company, J. Lancis, and P. Andrés, Prog. Opt. 56, 1 (2011).

[28] R. Salem, M. A. Foster, and A. L. Gaeta, Adv. Opt. Photonics 5, 274 (2013).

[29] L. Cohen, Time-Frequency Analysis (Prentice-Hall, New York, 1995).

[30] S. Qian and D. Chen, IEEE Signal Process. Lett. 16, 52 (1999).

[31] E. Sejdić, I. Djurović, and J. Jiang, Digit. Signal Process. 19, 153 (2009).

[32] S. Mallat, A Wavelet Tour of Signal Processing, 3rd ed. (Elsevier, New York, 2009).

[33] I. Juvells, A. Carnicer, J. Ferré-Borrull, E. Martín-Badosa, and M. Montes-Usategui, Eur. J. Phys. 27, 1111 (2006). 
[34] B. Lamine, C. Fabre, and N. Treps, Phys. Rev. Lett. 101, 123601 (2008).

[35] V. Giovannetti, S. Lloyd, and L. Maccone, Nat. Photonics 5, 222 (2011).

[36] S. Wang, X. Xiang, N. Treps, C. Fabre, R. Dong, T. Liu, and S. Zhang, Sci. Adv. 4, eaap9416 (2018).

[37] A. Lyons, G. C. Knee, E. Bolduc, T. Roger, J. Leach, E. M. Gauger, and D. Faccio, arXiv:1708.08351.

[38] R. Kaltenbaek, J. Lavoie, D. N. Biggerstaff, and K. J. Resch, Nat. Phys. 4, 864 (2008).

[39] P. Jian, O. Pinel, C. Fabre, B. Lamine, and N. Treps, Opt. Express 20, 27133 (2012).

[40] V. Thiel, J. Roslund, P. Jian, C. Fabre, and N. Treps, Q. Sci. Tech. 2, 034008 (2017).

[41] D. Bradley, B. Liddy, and W. Sleat, Opt. Commun. 2, 391 (1971).

[42] J. Rehacek, Z. Hradil, B. Stoklasa, M. Paúr, J. Grover, A. Krzic, and L. L. Sánchez-Soto, Phys. Rev. A 96, 062107 (2017).

[43] A. Eckstein, B. Brecht, and C. Silberhorn, Opt. Express 19, 13770 (2011).

[44] P. Manurkar, N. Jain, M. Silver, Y.-P. Huang, C. Langrock, M. M. Fejer, P. Kumar, and G. S. Kanter, Optica 3, 1300 (2016).

[45] D. V. Reddy and M. G. Raymer, Opt. Express 25, 12952 (2017).
[46] V. Ansari, G. Harder, M. Allgaier, B. Brecht, and C. Silberhorn, Phys. Rev. A 96, 063817 (2017).

[47] V. Ansari, J. M. Donohue, B. Brecht, and C. Silberhorn, Optica 5, 534 (2018).

[48] See Supplemental Material at http://link.aps.org/ supplemental/10.1103/PhysRevLett.121.090501 for mathematical details concerning the quantum pulse gate measurement and the tomographic methods employed in this Letter.

[49] M. Allgaier, V. Ansari, L. Sansoni, C. Eigner, V. Quiring, R. Ricken, G. Harder, B. Brecht, and C. Silberhorn, Nat. Commun. 8, 14288 (2017).

[50] J. Huang, X. Xie, C. Langrock, R. Roussev, D. Hum, and M. Fejer, Opt. Lett. 31, 604 (2006).

[51] D. V. Reddy and M. G. Raymer, Optica 5, 423 (2018).

[52] K. A. G. Fisher, D. G. England, J.-P. W. MacLean, P. J. Bustard, K. J. Resch, and B. J. Sussman, Nat. Commun. 7, 11200 (2016).

[53] J. H. Munns, S. Park, B. Brecht, and I. A. Walmsley, Frontiers in Optics (Optical Society of America, Washington, 2017), pp. JW3A-34.

[54] C. Polycarpou, K. N. Cassemiro, G. Venturi, A. Zavatta, and M. Bellini, Phys. Rev. Lett. 109, 053602 (2012).

[55] J. Roslund, R. M. De Araujo, S. Jiang, C. Fabre, and N. Treps, Nat. Photonics 8, 109 (2014). 